\title{
Relationship between Neural Activation and Electric Field Distribution during Deep Brain Stimulation
}

\author{
Mattias Åström, Elin Diczfalusy, Hubert Martens and Karin Wårdell
}

\section{Linköping University Post Print}

\section{Tweet}

N.B.: When citing this work, cite the original article.

Mattias Åström, Elin Diczfalusy, Hubert Martens and Karin Wårdell, Relationship between Neural Activation and Electric Field Distribution during Deep Brain Stimulation, 2015, IEEE Transactions on Biomedical Engineering, (62), 2, 664-72.

http://dx.doi.org/10.1109/TBME.2014.2363494

C2015 IEEE. Personal use of this material is permitted. However, permission to reprint/republish this material for advertising or promotional purposes or for creating new collective works for resale or redistribution to servers or lists, or to reuse any copyrighted component of this work in other works must be obtained from the IEEE.

Postprint available at: Linköping University Electronic Press

http://urn.kb.se/resolve?urn=urn:nbn:se:liu:diva-113588 


\title{
Relationship between Neural Activation and Electric Field Distribution during Deep Brain Stimulation
}

\author{
Mattias Åström, Elin Diczfalusy, Hubert Martens and Karin Wårdell, Member, IEEE
}

\begin{abstract}
Models and simulations are commonly used to study deep brain stimulation (DBS). Simulated stimulation fields are often defined and visualized by electric field isolevels or volumes of tissue activated (VTA). The aim of the present study was to evaluate the relationship between stimulation field strength as defined by the electric potential, $V$, the electric field, $E$, and the divergence of the electric field $\nabla^{2} V$, and neural activation. Axon cable models were developed and coupled to finite element DBS models in 3D. Field thresholds $\left(V_{T}, E_{T}\right.$, and $\left.\nabla^{2} V_{T}\right)$ were derived at the location of activation for various stimulation amplitudes ( 1 to $5 \mathrm{~V}$ ), pulse widths ( 30 to $120 \mu \mathrm{s}$ ), and axon diameters $(2.0$ to $7.5 \mu \mathrm{m})$. Results showed that thresholds for $V_{T}$ and $\nabla^{2} V_{T}$ were highly dependent on the stimulation amplitude while $E_{T}$, were approximately independent of the amplitude for large axons. The activation field strength thresholds presented in this study may be used in future studies to approximate the VTA during model-based investigations of DBS without the need of computational axon models.
\end{abstract}

Index Terms - axon cable model, deep brain stimulation (DBS), finite element method (FEM), simulation, field visualization

\section{INTRODUCTION}

$\mathrm{D}$ EEP brain stimulation (DBS) is an effective treatment for movement disorders such as Parkinson's disease, essential tremor and dystonia [1, 2]. DBS leads are implanted with stereotactic neurosurgical techniques in the deep regions of the brain [3]. Chronic electrical stimulation is delivered to the leads from battery-operated pulse generators that are implanted below the clavicle. The clinical benefit of DBS is largely dependent on the spatial distribution of the stimulation field in relation to brain anatomy [4-8].

This work was supported by the European Union's Seventh Framework Programme IMPACT (Grant agreement no: 305814) and by the Swedish Research Council (Grant No. 621-2013-6078).

M. Aström is with the Department of Biomedical Engineering, Linköping University, Linköping, Sweden and Sapiens Steering Brain Stimulation B.V., Eindhoven, The Netherlands (e-mail: matas@imt.liu.se).

E. Diczfalusy is with the Department of Biomedical Engineering, Linköping University, Linköping, Sweden (email: elidi@imt.liu.se).

H. Martens is with Sapiens Steering Brain Stimulation B.V., Eindhoven, The Netherlands (email: hubert.martens@ sapiensneuro.com).

K. Wårdell is with the Department of Biomedical Engineering, Linköping University, Linköping Sweden (e-mail: karin.wardell@liu.se, corresponding author)

Copyright (c) 2014 IEEE. Personal use of this material is permitted. However, permission to use this material for any other purposes must be obtained from the IEEE by sending an email to pubs-permissions@ieee.org
To maximize therapeutic benefits while avoiding unwanted side-effects, precise control over the stimulation field is essential.

Finite element models and simulations of DBS are commonly used to calculate and display the distribution of the stimulation field. Stimulation fields may be represented and visualized by different electrical quantities such as the electric potential $[6,9,10]$, the electric field [11-13], the second difference of the electric potential, $[4,14,15]$, as well as with a volume of tissue activated (VTA) derived from neuron models coupled to finite element simulations $[6,8,16,17]$. During modelling and simulation of DBS a challenge is to display a stimulation field that is relevant to the stimulationinduced therapeutic effects as well as side-effects. In order to visualize an electric entity that is relevant for the therapeutic outcome of DBS it should ideally be related to what is responsible for the clinical effects. However, the mechanisms of DBS are not fully known. It is hypothesized that jamming of the pathological activity is likely to play a prominent role [18]. It is further hypothesized that activation of axons may to a large part be responsible for the jamming but also for inducing stimulation-induced side-effects [19].

Activation of axons during extracellular stimulation is, however, rather complex. First of all, the threshold for activation is widely ranging for different sizes of axons [20], and it is not known what sizes of axons are responsible for therapeutic effects and side-effects during DBS. In addition, polarization at a particular axon node (voltage sensitive ion channel) is dependent on the potential differences at the adjacent nodes on either side of the particular node [21]. Thus, it is not possible to derive the amount of polarization at a specific node without considering the field distribution in the vicinity of that location i.e. the curvature of the stimulation field. In addition, as presented in Weiss' equation [22] the width of the stimulation pulse affects the polarization of neurons in a non-linear fashion.

Activation of neurons of a specific type, size and orientation in relation to the stimulation field may be investigated by the coupling of neuron compartment models to finite element simulations [4, 14, 17, 23, 24]. Simulations with coupled neuron models are, however, limited by their complexity, and the extensive execution time, as well as the obvious reason that it is not known what neurons are responsible for certain effects and side-effects. The complexity of implementing and solving axon models may be a major hurdle for many research groups that would like to perform model-based investigations 
of DBS. Computational time is also a major issue for software tools that are to be used in clinical practice. In the present study we sought to identify activation threshold levels related to the simulated stimulation field that can be used to define the VTA. Specifically, the aim was to derive activation threshold levels for the electric potential, the electric field, and the second derivative of the electric potential (activating function) and investigate if these can be used to define the VTA during model-based investigations of DBS, without the need for computational axon models.

\section{METHOD}

\section{A. Axon model}

A computational axon cable model was developed to define axonal activation in response to DBS stimulation. The electrophysiological response of the axon was modelled based on ion channel kinetics and channel densities as described by Wesselink et al. [25] and Richardson et al. [26]. The general equation describing the electrical response of the neuron is given by:

$$
\mathrm{c}_{n} \frac{d V_{m, n}}{d t}+\sum_{x} g_{x}\left(V_{m}-v_{x}\right)-g_{A} \cdot \Delta^{2} V_{m, n}=g_{A} \cdot \Delta^{2} V_{\mathrm{e}, \mathrm{n}}
$$

Where the first term on the left-hand side describes the capacitive membrane current proportional to the total membrane capacitance $c_{n}$ at each position $n$ along the fiber over time; $V_{m, n}$ corresponds to the membrane potential at position $n$. The second term represents the ionic membrane conductance at each position; fast potassium, slow potassium, fast sodium and leak conductance at nodes of Ranvier and leak conductance at myelinated internodes. The dynamics of the ionic conductance at each node of Ranvier were modelled according to experimental data reported for human fibers [27]. The third term gives the axial current flow which is proportional to the axial conductance $g_{A}$ between successive fiber segments and the second spatial difference of the membrane potential $\Delta^{2} V_{\mathrm{m}, \mathrm{n}}$. The right-hand part of the equation describes the driving term due to the extracellular electrical potential $V_{\mathrm{e}}$; this driving term is often referred to as the activating function (AF). The $\mathrm{AF}$ is proportional to the discretized second spatial derivative of $V_{e}$ along the nodes of the axon, according to:

$$
\Delta^{2} V_{e}(\mathrm{n})=V_{e}(\mathrm{n}-1)+V_{e}(\mathrm{n}+1)-2 V_{e}(\mathrm{n})
$$

In addition, the internodal myelinated portion of the fibres was explicitly modelled in order to match empirical data with regards to conduction velocity and strength-duration. The fibre geometry also plays an important part in the axon cable model, since it strongly affects physiological properties such as the action potential conduction velocity and the sensitivity to extra cellular stimuli [21]. In the current model, the fibre geometry was defined by its internodal length $L(\mathrm{~mm})$, the outer diameter $D(\mu \mathrm{m})$, the internodal axon inner diameter $d(\mu \mathrm{m})$

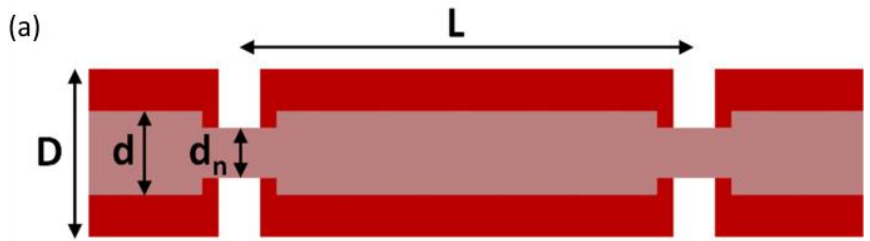

(b)

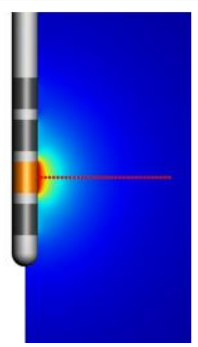

(c)

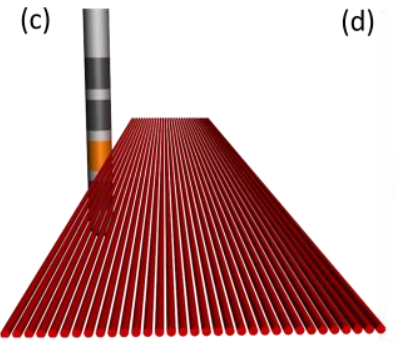

(d)

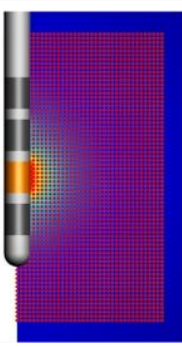

Fig. 1. (a) Parameters defining the geometry of the axon cable model where $D$ is the outer diameter, $d$ the inner diameter, $d_{n}$ the nodal diameter, and $L$ the internodal distance. (b) and (c) DBS lead with simulated electric potential together with red markers displaying the locations of the axon cable model. Here the axon models were located in a plane at the level of the active contact. (d) DBS lead together with red markers displaying the locations of the axon cable model in planes at multiple locations along the lead. The axon cable models were oriented perpendicular to the DBS lead in all cases.

and the nodal axon diameter $d_{n}(\mu \mathrm{m})$ (Fig. 1a). The relation between $L$ and $d$ was approximated by a power law according to

where

$$
L=146 d^{1.12}
$$

$$
d=D * 0.74(1-\exp (-D / 1.15))
$$

This relationship was derived from morphometric data of mammalian CNS fibers [28-31] and is valid for $D$ in the interval 1.5 to $10 \mu \mathrm{m}$.

Definitions of the nodal parameters used in the model are presented in the Appendix, together with plots presenting the relation between $d, D$ and $L$ for the morphometric data used for construction of the axon model. Ionic membrane conductance at nodes of Ranvier were modelled based on data by Schwarz et al. [27] who studied human peripheral nerves (see Appendix). The leak conductance, $g_{L}$ at nodes of Ranvier and the fiber diameter $D$ are the only parameters that were varied in order to match the fiber model to available data for clinical DBS data. All the other parameters were obtained from experimental literature. In order to optimize $g_{L}$, computed chronaxies were matched to strength-duration data for stimulation in ventral intermedius nucleus of thalamus and the internal segment of the globus pallidus (GPi) [32, 33]. Relevant fiber-diameter range for clinical DBS was estimated by matching simulation results to threshold-distance data for thalamic DBS [34].

Axon cable models with 21 nodes were constructed for a range of different axon diameters (2.0 to $7.5 \mu \mathrm{m})$ corresponding to a range in intermodal length of 0.182 to $0.994 \mathrm{~mm}[31,35]$. The axon models were implemented in MatLab (The MathWorks, USA), and the solver 'ode15' was used to integrate the differential equations describing each axon cable model. 


\section{B. DBS model}

A model of the DBS lead as well as surrounding tissue was set up and used for simulation of the stimulation field. The DBS lead was modelled with a diameter of $1.27 \mathrm{~mm}, 4$ electrode contacts with a height of $1.5 \mathrm{~mm}$ separated by 0.5 $\mathrm{mm}$ in order to mimic Medtronic lead model 3389, (Medtronic Inc., USA). The surrounding tissue was modelled as a sphere with a radius of $30 \mathrm{~cm}$ placed as the outer boundary from the active electrode contact. Homogeneous brain tissue was assumed for the sphere and the layer of distributed resistance, and was modelled with a conductivity $\sigma=0.1 \mathrm{~S} / \mathrm{m}$. Simulations were carried out during single contact monopolar settings where contact 1 was active and the outer boundary of the tissue was set to ground. Non active contacts were set to floating [36]. The total impedance of the model during single contact monopolar settings was $1 \mathrm{k} \Omega$. Simulations were carried out with COMSOL Multiphysics 4.4 (COMSOL AB, Stockholm, Sweden) and consisted of approximately 500,000 tetrahedral mesh elements. The mesh density was defined by the built in physics-controlled mesh generator, where the smallest elements were located by the electrode contacts in order to capture the strong electric field gradients by the edges. The distribution of the electric potential, $V_{e}$, in the tissue was simulated for various DBS amplitudes $\left(V_{D B S}\right)$ by solving the Laplace equation for steady currents [37]:

$$
\nabla \cdot \overrightarrow{\mathrm{J}}=-\nabla \cdot\left[\sigma \nabla \mathrm{V}_{e}\right]=0
$$

For each amplitude, the distribution of the electric potential was sampled in a region of interest and exported to MatLab. The sampled region of interest was defined as a plane at the level of the active contact, perpendicular to the lead (XYplane) with a radial extension of 0 to $7 \mathrm{~mm}$ in $\mathrm{X}$, and -20 to 20 $\mathrm{mm}$ in $\mathrm{Y}$.

\section{Simulations}

By combining the constructed axon cable models, the exported field distributions, and a function that defined the stimulation waveform over one stimulation cycle, the axonal responses to extracellular stimulation were computed. The function defining the stimulation waveform was based on output from the Medtronics Itrel II device [38], and consisted of a cathodic-first biphasic charge-balanced waveform with a $0.4 \mathrm{~ms}$ interphase gap. The width of the cathodic pulse was related to the pulse width, while the anodic counter-pulse width was set to 10 times that of the cathodic pulse with an amplitude 0.1 times the cathodic pulse amplitude. The cathodic pulse amplitude was defined as the cathodic-peak to anodic-peak voltage of the DBS waveform.

Axon models were positioned perpendicular to the lead within the sampled region of interest at a radial distance of 0.7 to 7 $\mathrm{mm}$, in steps of $0.1 \mathrm{~mm}$ from the center of the DBS lead (Fig. $1 \mathrm{~b}$ and Fig. 1c). For each stimulation configuration the maximum radial distance for which the axon was activated was stored. At this location of activation the field strength was measured. The field strength was measured in three different entities; the electric potential $\left(V_{T}\right)$, the magnitude of the
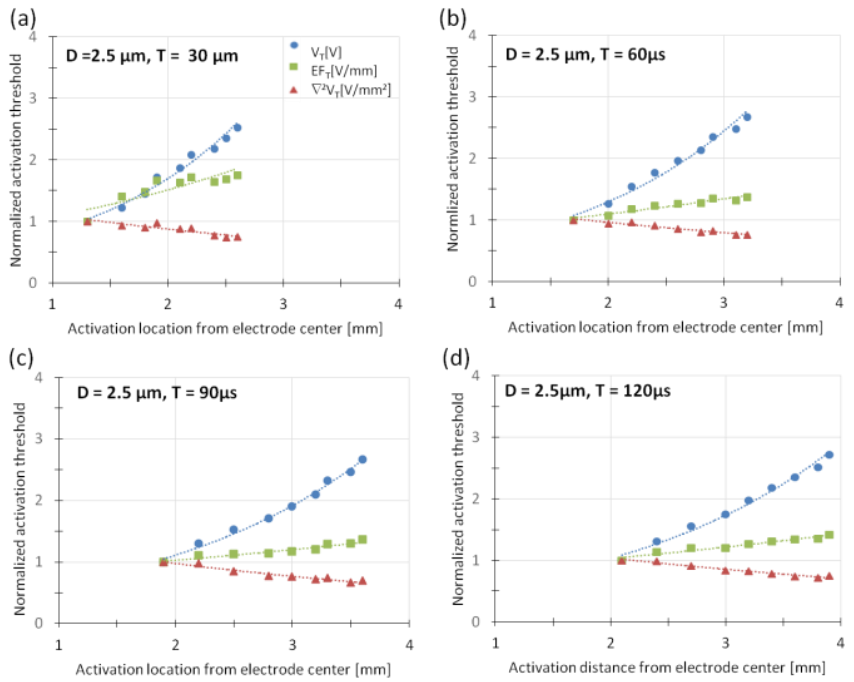

Fig. 2. Normalized simulated activation field thresholds, $V_{T}, E_{T}, \nabla^{2} V_{T}$, for nine stimulation amplitudes $\left(V_{D B S}=1.0\right.$ to $5 \mathrm{~V}$, in steps of $\left.0.5 \mathrm{~V}\right)$, four pulse widths ( $T=30,60,90$ and $120 \mu \mathrm{s}$ ), and a fiber diameter, $D$, of $2.5 \mu \mathrm{m}$.

gradient of the electric potential $\left(\nabla V_{T}\right)$ commonly referred to as the electric field strength $\left(E_{T}\right)$, and the divergence of the electric field $\left(\nabla^{2} V_{T}\right)$ which is a generalization of the second derivative of the electric potential in three dimensions. In total, 288 simulations were carried out for the fiber diameters, $D$, (2.0 2.5, 3.0, 3.5, 4.0, 4.5, 5.0, and 7.5 $\mu \mathrm{m})$, pulse widths, $T$, $(30,60,90$, and $120 \mu \mathrm{s})$, and stimulation amplitudes, $V_{D B S}$, $(1.0$ to $5.0 \mathrm{~V}$; in steps of $0.5 \mathrm{~V})$. In order to further investigate the relationship between axonal activation and $V_{T}, E_{T}$, and $\nabla^{2} V_{T}$ additional simulations were carried out for axon diameters of 2.5, 5.0, and $7.5 \mu \mathrm{m}$, during stimulation with $V_{D B S}$ of $3 \mathrm{~V}$, and a pulse width set to $60 \mu \mathrm{s}$. Axons were then located in the vicinity of the DBS lead similar to the previously explain simulation set up, but in multiple planes separated by $0.1 \mathrm{~mm}$ to cover the full field along the lead (Fig. 1d). The result was visualized with stimulation fields for each entity $\left(V_{T}, E_{T}\right.$, and $\left.\nabla^{2} V_{T}\right)$ as derived at the level of the active contact, together with red markers at the locations where the axons were activated. (a)

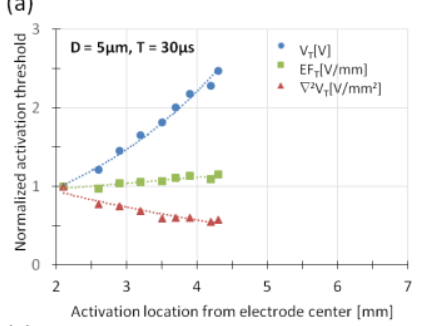

(c)

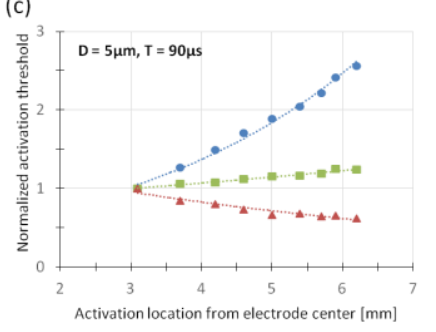

(d)
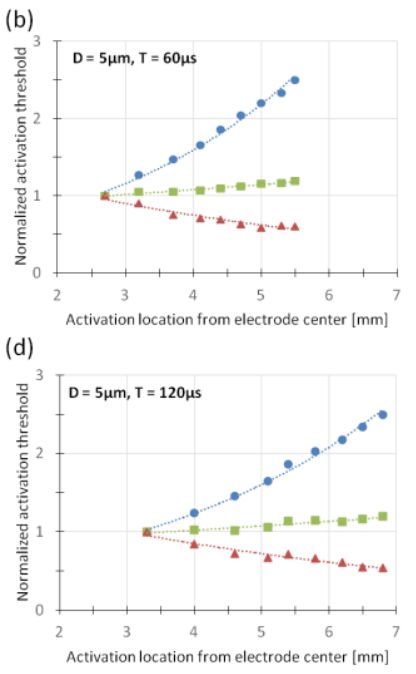

Fig. 3. Normalized simulated activation field thresholds, $V_{T}, E_{T}, \nabla^{2} V_{T}$, for nine stimulation amplitudes $\left(V_{D B S}=1.0\right.$ to $5 \mathrm{~V}$, in steps of $\left.0.5 \mathrm{~V}\right)$, four pulse widths ( $T=30,60,90$ and $120 \mu \mathrm{s}$ ), and a fiber diameter, $D$, of $5.0 \mu \mathrm{m}$. 
(a)

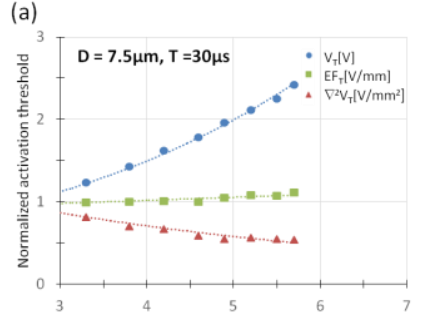

(c)

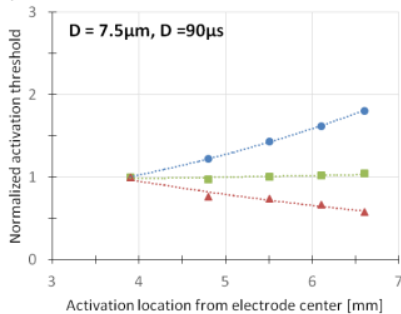

(b)

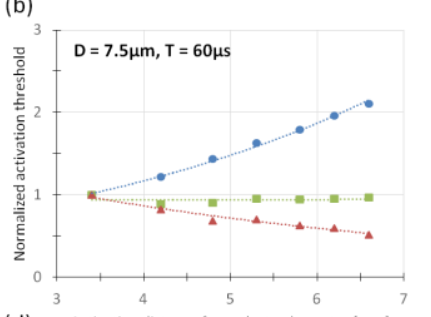

(d) Activation distance from electrode center $[\mathrm{mm}]$

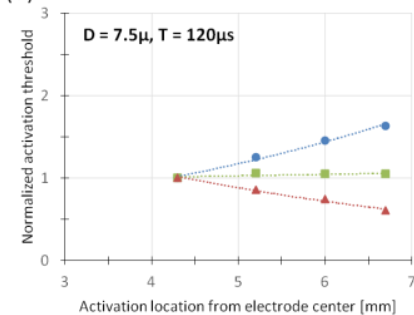

Fig. 4. Normalized simulated activation field thresholds, $V_{T}, E_{T}, \nabla^{2} V_{T}$, for nine stimulation amplitudes $\left(V_{D B S}=1.0\right.$ to $5 \mathrm{~V}$, in steps of $\left.0.5 \mathrm{~V}\right)$, four pulse widths ( $T=30,60,90$ and $120 \mu \mathrm{s}$ ), and a fiber diameter, $D$, of $7.5 \mu \mathrm{m}$.

\section{Data analysis}

Activation field thresholds $\left(V_{T}, E_{T}\right.$ and $\left.\nabla^{2} V_{T}\right)$ at the location of activation were derived for all stimulation configurations. Median field thresholds (median) and range (min-max) were calculated for each axon diameter and pulse width. In order to compare the activation field thresholds for each entity $\left(V_{T}, E_{T}\right.$, and $\left.\nabla^{2} V_{T}\right)$ they were normalized with the activation field strength derived during the lowest stimulation amplitude setting i.e. $V_{D B S}=1.0 \mathrm{~V}$.

\section{E. Comparison with other models}

In order to relate our results to existing DBS models our results were compared with voltage-distance data from a widely used axon model developed by McIntyre et al. [4, 39]. Their voltage-distance data was derived during stimulation with a $T=100 \mu$ s and $D=5.7 \mu \mathrm{m}$. The axon diameter of our model was adjusted in order to align with their results.

In addition, our derived activation threshold levels were compared with two previous studies were neural activation during DBS was investigated [34, 40]. In the study by Kuncel et al. [34] the spatial extent of activation in thalamic DBS during stimulation with a $T=90 \mu$ s was investigated. When compared with our results their derived relationship was implemented as:

$$
r(\mathrm{U})=\sqrt{\frac{U-U_{\text {offset }}}{k}}
$$

Where $U_{\text {offset }}=0.1$, and $k=0.22$.

In addition, comparison were carried out with the results of Madler and Coenen [40] who presented a function that describes the radius of the volume of activated tissue during stimulation with $T=60 \mu$ s in the subthalamic nucleus target area:

$$
r(\Omega, \mathrm{U})=-\frac{k_{4} \Omega-\sqrt{\mathrm{k}_{4}^{2} \Omega^{2}+2 k_{1} k_{4} \Omega+\mathrm{k}_{1}^{2}+4 k_{3} U+k_{1}}}{2 k_{3}}
$$

Where $r$ is the radius of the VTA, $\Omega$ the tissue impedance, $U$ the stimulation amplitude in volt, and $k_{l}, k_{3}$, and $k_{4}$ the derived constants. When compared to our results the following constants were applied:

$$
\begin{gathered}
k_{l}=-1.0473, k_{3}=0.2786, k_{4}=0.0009856 \text {, and } \Omega=1000 \\
\text { III. RESULTS }
\end{gathered}
$$

\section{A. Activation field thresholds}

Activation field thresholds were derived for $V_{T}, E_{T}$, and $\nabla^{2} V_{T}$ for all simulated axon diameters, pulse widths, and $V_{D B S}$ settings. The activation field thresholds were normalized for comparison between the different entities. Normalized results for the axon diameters 2.5, 5.0, and $7.5 \mu \mathrm{m}$ and the different pulse width are presented in Fig. 2, Fig. 3 and Fig. 4. Activation field thresholds for the electric potential, $V_{T}$ increased substantially along with an increased $V_{D B S}$. The influence of the amplitude was most pronounced for the smallest fiber diameter and shortest pulse width (Fig. 2). Activation field thresholds for the electric field, $E_{T}$, increased with increased amplitude for the smallest fiber diameter, but remained approximately constant for larger fibers $(D=5 \mu \mathrm{m}$ and $7.5 \mu \mathrm{m}$ ) (Fig. 3 to 4 ). Activation field thresholds for $\nabla^{2} V_{T}$ decreased with increased stimulation amplitude for all fiber diameters and pulse widths. Median and range of the normalized activation field thresholds for $V_{T}, E_{T}$, and $\nabla^{2} V_{T}$ during stimulation with $T=60 \mu$ s are presented in Table 1 . For large axons $(5.0$ and $7.5 \mu \mathrm{m})$ the median of $E_{T}$ was close to 1 with a relatively small range (1.00 to 1.19$)$ and $(0.97$ to 1.06) respectively, while $V_{T}$ and $\nabla^{2} V_{T}$ showed larger ranges. Absolute activation field thresholds for $V_{T}, E_{T}$, and $\nabla^{2} V_{T}$ are presented in Table 2.

TABLE 1

NORMALISED ACTIVATION FIELD THRESHOLDS OF THE ELECTRIC POTENTIAL $V_{T}(\mathrm{~V})$, THE ELECTRIC FIELD $E_{T}(\mathrm{~V} / \mathrm{MM})$, AND THE DIVERGENCE OF THE ELECTRIC FIELD $\nabla^{2} V_{T}\left(\mathrm{~V} / \mathrm{MM}^{2}\right)$ FOR THE THREE DIFFERENT AXON DIAMETERS, $D$, AT A PULSE WIDTH OF $60 \mu \mathrm{S}$. ACTIVATION FIELD THRESHOLDS WERE DERIVED FOR AMPLITUDES, $V_{D B S}$, OF 1 TO 5 V. NORMALISATION WAS PERFORMED WITH ACTIVATION FIELD THRESHOLDS FOR THE LOWEST, $V_{D B S}$, VALUE I.E. $1 \mathrm{~V}$.

\begin{tabular}{cccccccccc}
\hline \hline \multicolumn{1}{c}{$D=2.5 \mu m$} & \multicolumn{4}{c}{$D=5.0 \mu m$} & \multicolumn{3}{c}{$D=7.5 \mu m$} \\
$\boldsymbol{V}_{D B S}$ & $\boldsymbol{V}_{\boldsymbol{T}}$ & $\boldsymbol{E}_{\boldsymbol{T}}$ & $\nabla^{2} \boldsymbol{V}_{\boldsymbol{T}}$ & $\boldsymbol{V}_{\boldsymbol{T}}$ & $\boldsymbol{E}_{\boldsymbol{T}}$ & $\boldsymbol{\nabla}^{2} \boldsymbol{V}_{\boldsymbol{T}}$ & $\boldsymbol{V}_{\boldsymbol{T}}$ & $\boldsymbol{E}_{\boldsymbol{T}}$ & $\boldsymbol{\nabla}^{2} \boldsymbol{V}_{\boldsymbol{T}}$ \\
\hline 1 & 1.00 & 1.00 & 1.00 & 1.00 & 1.00 & 1.00 & 1.00 & 1.00 & 1.00 \\
1.5 & 1.27 & 1.07 & 0.94 & 1.27 & 1.05 & 0.90 & 1.22 & 0.97 & 0.82 \\
2 & 1.55 & 1.18 & 0.96 & 1.47 & 1.05 & 0.75 & 1.43 & 0.99 & 0.69 \\
2.5 & 1.78 & 1.23 & 0.92 & 1.66 & 1.07 & 0.71 & 1.63 & 1.04 & 0.70 \\
3 & 1.97 & 1.26 & 0.85 & 1.86 & 1.10 & 0.69 & 1.79 & 1.03 & 0.63 \\
3.5 & 2.14 & 1.28 & 0.80 & 2.04 & 1.12 & 0.63 & 1.96 & 1.04 & 0.59 \\
4 & 2.36 & 1.36 & 0.83 & 2.20 & 1.15 & 0.59 & 2.11 & 1.06 & 0.52 \\
4.5 & 2.48 & 1.33 & 0.76 & 2.34 & 1.17 & 0.61 & - & - & - \\
5 & 2.68 & 1.37 & 0.76 & 2.50 & 1.19 & 0.61 & - & - & - \\
\hline Min & 1.00 & 1.00 & 0.76 & 1.00 & 1.00 & 0.59 & 1.00 & 0.97 & 0.52 \\
Median & 1.97 & 1.26 & 0.85 & 1.86 & 1.10 & 0.69 & 1.63 & 1.03 & 0.69 \\
Max & 2.68 & 1.37 & 1.00 & 2.50 & 1.19 & 1.00 & 2.11 & 1.06 & 1.00 \\
\hline \hline
\end{tabular}


As expected it was found that the activation field thresholds for all electrical entities were lower for larger fiber diameters and for longer pulse widths. In order to cover the range of clinically relevant axon diameters in DBS targets, additional simulations were carried out for axons in the range 2.0 to 5.0 $\mu \mathrm{m}$ in steps of $0.5 \mu \mathrm{m}$ (Tab. 3).

Comparisons between $V_{T}, E_{T}$, and $\nabla^{2} V_{T}$ and axonal activation were also carried out at different depths along the lead for axon diameters of 2.5, 5.0, and $7.5 \mu \mathrm{m}$. The results was visualized with stimulation field isolevels for each entity $\left(V_{T}, E_{T}\right.$, and $\left.\nabla^{2} V_{T}\right)$ at thresholds derived at the level of the active contact. Red markers displays the locations where action potentials was initiated (Fig. 5). It was found that all the electrical entities roughly fitted the volume of axonal activation, although none of the electrical entities aligned perfectly with the VTA.

\section{B. Comparison with other models}

The results from our axon model were compared with voltage-distance data from McIntyre's axon model (Fig. 6a). In order to align the results a pulse width of $100 \mu$ s and an axon diameter of $3.5 \mu \mathrm{m}$ were used for our axon model, contrary to what was used in McIntyre's study $5.7 \mu \mathrm{m}$ and 100 $\mu \mathrm{s}$. The difference in axon diameter was to a large part related to how the two models calculated the diameter based internodal length. After this adjustment both models showed similar results. In Fig. 6b two activation field thresholds for the electric field $(0.165 \mathrm{~V} / \mathrm{mm}$ and $0.190 \mathrm{~V} / \mathrm{mm})$ were used for comparison with the results of Kuncel et al. and Madler and Coenen. The electric field matched well with both studies.

TABLE 2 .

ABSOLUTE ACTIVATION FIELD THRESHOLDS OF THE ELECTRIC POTENTIAL $V_{T}$ $(\mathrm{V})$, THE ELECTRIC FIELD $E_{T}$ (V/MM), AND THE DIVERGENCE OF THE ELECTRIC FIELD $\nabla^{2} V_{T}\left(\mathrm{~V} / \mathrm{MM}^{2}\right)$ FOR THREE DIFFERENT AXON DIAMETERS $D(\mu M)$, AT A STIMULATION PULSE WIDTH $T$ OF $60 \mu \mathrm{S}$. ACTIVATION FIELD THRESHOLDS WERE DERIVED FOR DIFFERENT AMPLITUDES $V_{D B S}(\mathrm{~V})$.

\begin{tabular}{ccccccccccc}
\hline \hline & \multicolumn{4}{c}{$D=2.5 \mu m$} & \multicolumn{3}{c}{$D=5.0 \mu m$} & \multicolumn{3}{c}{$D=7.5 \mu m$} \\
$V_{D B S}$ & $\boldsymbol{V}_{\boldsymbol{T}}$ & $\boldsymbol{E}_{\boldsymbol{T}}$ & $\boldsymbol{\nabla}^{2} \boldsymbol{V}_{\boldsymbol{T}}$ & $\boldsymbol{V}_{\boldsymbol{T}}$ & $\boldsymbol{E}_{\boldsymbol{T}}$ & $\boldsymbol{\nabla}^{2} \boldsymbol{V}_{\boldsymbol{T}}$ & $\boldsymbol{V}_{\boldsymbol{T}}$ & $\boldsymbol{E}_{\boldsymbol{T}}$ & $\boldsymbol{\nabla}^{2} \boldsymbol{V}_{\boldsymbol{T}}$ \\
\hline 1 & 0.428 & 0.255 & 0.362 & 0.271 & 0.100 & 0.921 & 0.216 & 0.062 & 0.448 \\
1.5 & 0.544 & 0.274 & 0.342 & 0.344 & 0.105 & 0.831 & 0.264 & 0.061 & 0.369 \\
2 & 0.662 & 0.301 & 0.349 & 0.398 & 0.106 & 0.692 & 0.309 & 0.062 & 0.307 \\
2.5 & 0.761 & 0.315 & 0.332 & 0.450 & 0.107 & 0.656 & 0.351 & 0.065 & 0.314 \\
3 & 0.844 & 0.323 & 0.309 & 0.504 & 0.110 & 0.634 & 0.386 & 0.064 & 0.281 \\
3.5 & 0.914 & 0.326 & 0.290 & 0.553 & 0.112 & 0.578 & 0.423 & 0.065 & 0.266 \\
4 & 1.009 & 0.346 & 0.301 & 0.595 & 0.115 & 0.544 & 0.455 & 0.066 & 0.232 \\
4.5 & 1.063 & 0.338 & 0.277 & 0.632 & 0.117 & 0.565 & - & - & - \\
5 & 1.146 & 0.351 & 0.277 & 0.677 & 0.119 & 0.559 & - & - & - \\
\hline \multirow{2}{*}{ Min } & 0.428 & 0.255 & 0.277 & 0.271 & 0.100 & 0.544 & 0.216 & 0.061 & 0.232 \\
Median & 0.844 & 0.323 & 0.309 & 0.504 & 0.110 & 0.634 & 0.351 & 0.064 & 0.307 \\
Max & 1.146 & 0.351 & 0.362 & 0.677 & 0.119 & 0.921 & 0.455 & 0.066 & 0.448 \\
\hline \hline
\end{tabular}

\section{DISCUSSION}

Finite element DBS models were coupled to a novel axon model in order to investigate the relationship between neural activation and the stimulation field strength at the location of activation. The results showed that the VTA can be approximated by a constant stimulation field threshold $\left(V_{T}, E_{T}\right.$, or $\left.\nabla^{2} V_{T}\right)$ presented in this study without the need to couple axon models to the finite element solution. The simulations were carried out for clinically relevant amplitudes and pulse widths typically used for treatment of Parkinson's disease [1].

TABLE 3 .

MEDIAN AND RANGE (MIN-MAX) OF THE ELECTRIC FIELD, $E_{T},(\mathrm{~V} / \mathrm{MM})$ ACTIVATION THRESHOLDS FOR VARIOUS PULSE WIDTHS $T(\mu \mathrm{S})$ AND AXON DIAMETERS $D(\mu \mathrm{M})$ IN STEPS OF $0.5 \mu \mathrm{M}$. SIMULATIONS WERE CARRIED OUT FOR AMPLITUDES OF 1 TO $5 \mathrm{~V}$ IN STEPS OF $0.5 \mathrm{~V}(\mathrm{~N}=9)$.

\begin{tabular}{ccccc}
\hline \hline & \multicolumn{4}{c}{ Pulse width, $\boldsymbol{T} \boldsymbol{\mu s}$} \\
$\boldsymbol{D}$ & 30 & 60 & 90 & 120 \\
$\boldsymbol{\mu m}$ & & & & \\
\hline & 0.765 & 0.457 & 0.376 & 0.323 \\
2.0 & $(0.556-0.833)$ & $(0.371-0.538)$ & $(0.288-0.433)$ & $(0,255-0.351)$ \\
& 0.504 & 0.323 & 0.240 & 0.210 \\
2.5 & $(0.421-0.538)$ & $(0.255-0.351)$ & $(0.205-0.279)$ & $(0.167-0.237)$ \\
& 0.376 & 0.240 & 0.185 & 0.157 \\
3.0 & $(0.322-0.401)$ & $(0.205-0.264)$ & $(0.150-0.202)$ & $(0.126-0.167)$ \\
& 0.300 & 0.185 & 0.142 & 0.121 \\
3.5 & $(0.255-0.316)$ & $(0.161-0.202)$ & $(0.126-0.154)$ & $(0.108-0.135)$ \\
& 0.240 & 0.150 & 0.115 & 0.096 \\
4.0 & $(0.226-0.264)$ & $(0.137-0.160)$ & $(0.100-0.130)$ & $(0.087-0.111)$ \\
& 0.225 & 0.142 & 0.107 & 0.090 \\
4.5 & $(0.205-0.237)$ & $(0.126-0.149)$ & $(0.093-0.119)$ & $(0.080-0.100)$ \\
& 0.177 & 0.110 & 0.087 & 0.074 \\
5.0 & $(0.161-0.192)$ & $(0.100-0.119)$ & $(0.075-0.094)$ & $(0.066-0.078)$ \\
\hline \hline
\end{tabular}

\section{A. Activation field thresholds}

Activation threshold levels for the electric potential, the electric field, and the divergence of the electric field, were derived at the location of activation during one contact monopolar stimulation. Simulations showed that the derived field thresholds were dependent upon stimulation amplitude, pulse width, and axon diameter. The electric field, $E_{T}$, showed the least sensitivity to stimulation amplitude, and were approximately independent of the amplitude for large axons (Fig. 2, Fig. 3, Fig. 4 and Table 1). This suggests that the VTA for large axons can be approximated by a constant electric field threshold independent of the stimulation amplitude, while for smaller axons the stimulation amplitude should be considered when using the electric field as an approximation of the VTA. The electric potential field thresholds, $V_{T}$, increased substantially with increased stimulation amplitude and showed a non-linear appearance. This has also been shown by Chaturverdi et al., [10]. The activation thresholds for the divergence of the electric field, $\nabla^{2} V_{T}$, decreased with increased stimulation amplitude. Similarly McIntyre et al. [4, 14] showed a non-linear decrease of the activation field strength by increased distance to axon for the second derivate of the electric potential.

Since the mechanisms of DBS are not well understood, it is not know which neurons are primarily responsible for the therapeutic effects and side effects. It is likely that certain side-effects are related to stimulation of large axons such as 

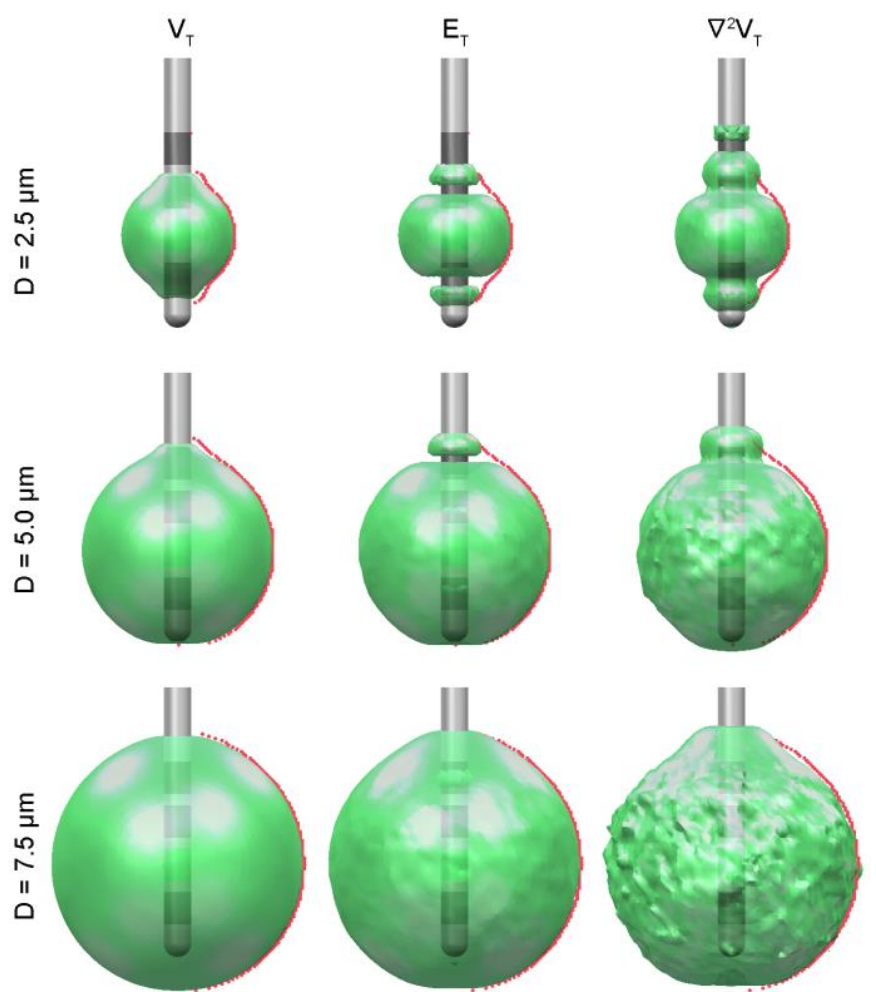

Fig. 5. Axonal activation in 3D for the electric potential $V_{T}$ the electric field $E_{T}$ and the second derivative of the electric potential $\nabla^{2} V_{T}$. The simulations were carried out for a stimulation amplitude $\mathrm{V}_{\mathrm{DBS}}$ of $3.0 \mathrm{~V}$, and pulse width $\mathrm{T}$ of $60 \mu \mathrm{s}$, and axon diameters D of $2.5 \mu \mathrm{m}, 5.0 \mu \mathrm{m}$, and $7.5 \mu \mathrm{m}$. The red markers represents the location where the axon cable models were activated.

capsular fibers, while other effects may be related to stimulation of smaller axons when nuclei are targeted such as the STN or the GPi. Measurements of axon diameters inside the STN and GPi have shown axon diameters ranging from 0.1 to $2.5 \mu \mathrm{m}$ [41]. In a model-based study axons in the STN were modelled with a diameter of $2 \mu \mathrm{m}$, while axons in the fields of Forel H2, substantial nigra, and zona incerta were modelled with a diameter of $3 \mu \mathrm{m}$ [24]. In order to cover this range of axon diameters additional simulations were also carried out and electric field thresholds were derived and presented in Table 3.

\section{B. Visualization}

Several previous studies have used an electric field isolevel of $0.2 \mathrm{~V} / \mathrm{mm}$ for defining the stimulation field [12, 13, 42]. In our study this isolevel correspond to a fiber diameter between 3 and $3.5 \mu \mathrm{m}$ for a pulse width of $60 \mu$ s (Table 3). When comparing the simulations with the results of Kuncel et al., and Madler and Coenen the $0.165 \mathrm{~V} / \mathrm{mm}$ and $0.190 \mathrm{~V} / \mathrm{mm}$ isolevels (Fig. 6b) would correspond to axon diameters between 3 and $4 \mu \mathrm{m}$. Multiple electric field isolevels have also been used to define and display the stimulation field [43, 44]. Such multilevel presentations might be useful in order to visualize tentative activation of different axons. Independent of the threshold chosen for visualization, the electric field has the advantage of making relative comparisons possible between patients and studies, since the results are not limited to a specific axon model.
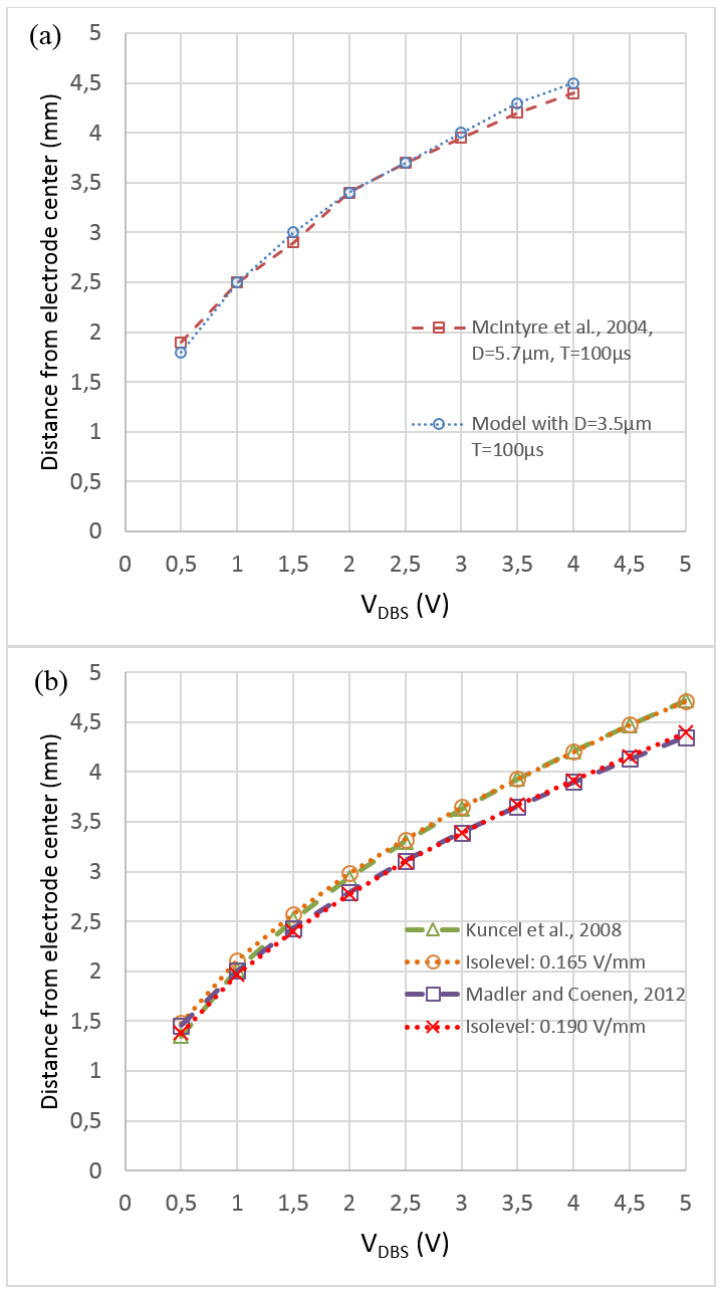

Fig. 6. (a) Comparison of our axon cable model with the axon cable model of McIntyre et al., 2004 [4]. In order to align the results a diameter of $3.5 \mu \mathrm{m}$ was used for our axon cable model. (b) Comparison between electric field strength and the results of Kuncel, and Madler and Coenen. [34, 40]. Electric field isolevels at 0.165 and $0.190 \mathrm{~V} / \mathrm{mm}$ correlated very well with the results of those studies.

\section{The axon model}

As the axon geometry and in particular the internodal length, $L$, has a substantial impact on the activation threshold, efforts were put to model $L$ based on detailed morphometric data previously reported in the literature [28-31]. From this data a power law (equation 3) was derived to calculate $L$ based on $D$. Resulting internodal lengths deviate slightly from the commonly approximated relationship of 100:1 [21]. The fit of the power law to the morphometric data is shown in Appendix Fig. 7.

Our axon model is valid for smaller fiber diameters (range 1.5 to $10 \mu \mathrm{m}$, see Appendix) than what is commonly used during model-based studies on DBS. This fiber diameter range should be compared to the neuron model presented by McIntyre et al., [39] which is valid for $D$ in the interval 5.7 to $16 \mu \mathrm{m}$, and commonly used in the model-based DBS community $[14,17,23,24]$. When comparing our axon model with this axon model it was clear that there was a difference with regards to axon diameters and corresponding internodal lengths (Fig. 6a). In McIntyre's model a $D$ of $5.7 \mu \mathrm{m}$ 
corresponds to an $L$ of $0.5 \mathrm{~mm}$, while in our model the same axon diameter corresponds to an $L$ of $0.726 \mathrm{~mm}$. Due to the substantial impact of the internodal length on the activation threshold it may be considered in the future to define modelled axons by their internodal length instead of their axon diameter.

\section{The DBS model}

Model-based studies are in general full of assumptions and simplifications [45, 46]. Thus, model-based investigations should always be considered on a rough level. Nevertheless, models and simulations can be valuable for providing knowledge on a general level, or when relative differences are studied. In the present study, homogeneous tissue was used in order to produce general results. Thus, no heterogeneity such as encapsulation tissue, tissue in-homogeneities, or anisotropic tissue properties were implemented although such properties will influence the results in the patient-specific case.

One contact cathodic monopolar stimulation settings was used together with a model of Medtronic lead model 3389. Thus, the results of this study are only valid for this case and it is not evident that the results can be generalized for other configurations and DBS leads. The underlying reason is that activation of axons is dependent upon the curvature of the stimulation field, and different configurations and DBS leads may generate fields with different field curvatures.

The curvature of the stimulation field may also be affected by the location of the grounded reference electrode as shown by Walckiers et al. 2010, [47] and the inclusion of a wholehead model [48]. Field thresholds for the electric field, $E_{T}$, showed the smallest influence of field curvatures with only a threshold range of $9 \%$ for the largest axon diameter $(7.5 \mu \mathrm{m})$ during stimulation with 1 to $5 \mathrm{~V}$ (Table 1 ). In order to minimize the influence of the curvature of the stimulation field, the electric field thresholds, $E_{T}$, may be preferred when approximating the VTA with a constant stimulation field threshold. Future studies may investigate how these activation thresholds are related to other modes of stimulation, such as double monopolar, bipolar, and tripolar stimulation, or stimulation with other DBS leads. In addition, patient specific tissue models with axons positioned in a patient-specific manner in the vicinity of the DBS lead have been used extensively in the past $[7,14,17,23]$. Similar studies could be carried out to investigate the impact of tissue heterogeneity [13] and anisotropy [44] on the stimulation field thresholds.

\section{CONCLUSION}

A neuron model valid for small axon diameters has been introduced and used together with finite element models of DBS for calculation of activation field thresholds. Results showed that the electric potential, the electric field as well as the second derivative of the electric potential can be used to approximate the VTA without the need to couple axon models to the finite element solution. Electric field thresholds showed the least sensitivity to stimulation amplitude, and were approximately independent of the amplitude for large axons. The activation field strength thresholds presented in this study may be used in future studies to approximately define the VTA during model-based investigations of DBS.

\section{APPENDIX}

\section{A. Axon model}

Ionic membrane conductance at nodes of Ranvier were modelled following data by [27] on human peripheral nerves. Nodal width was assumed $1 \mu \mathrm{m}$ independent of fiber diameter. Original data at $20^{\circ} \mathrm{C}$ have been corrected for $37^{\circ} \mathrm{C}$ using appropriate $\mathrm{Q}_{10}$ factors [25]. For clarity, units are given in behind the equations.

Transient $\mathrm{Na}^{+}$current:

$$
\begin{gathered}
i_{\mathrm{Na}}=m^{3} h p_{\mathrm{Na}} F^{2} \frac{V_{\mathrm{m}}}{R T} \frac{\left[\mathrm{Na}_{\mathrm{in}}\right]-\left[\mathrm{Na}_{\text {out }}\right] e^{-\left(F V_{\mathrm{m}} / R T\right)}}{1-e^{-\left(F V_{\mathrm{m}} / R T\right)}}\left(\mathrm{A} / \mathrm{m}^{2}\right) \\
\mathrm{d} m / \mathrm{d} t=\alpha_{\mathrm{m}}(1-m)-\beta_{\mathrm{m}} m(\mathrm{~Hz}) \\
\mathrm{d} h / \mathrm{d} t=\alpha_{\mathrm{h}}(1-h)-\alpha_{\mathrm{h}} h(\mathrm{~Hz}) \\
\alpha_{\mathrm{m}}=6.81 \times 10^{6} \times\left(V_{\mathrm{m}}+0.0184\right) /\left(1-e^{(-0.0184-V \mathrm{~m}) / 0.0103}\right)(\mathrm{Hz}) \\
\beta_{\mathrm{m}}=3.15 \times 10^{5} \times\left(-0.0227-V_{\mathrm{m}}\right) /\left(1-e^{(V \mathrm{~m}+0.0227) / 0.00916}\right)(\mathrm{Hz}) \\
\alpha_{\mathrm{h}}=2.17 \times 10^{5} \times\left(-0.111-V_{\mathrm{m}}\right) /\left(1-e^{(V \mathrm{~m}+0.111) / 0.0110}\right)(\mathrm{Hz}) \\
\beta_{\mathrm{h}}=1.48 \times 10^{4} /\left(1+\exp ^{(-0.0288-V \mathrm{~m}) / 0.0134}\right)(\mathrm{Hz})
\end{gathered}
$$

Fast $K^{+}$current:

$$
i_{K f}=g_{K f} n^{4}\left(V_{m}-E_{K}\right)\left(\mathrm{A} / \mathrm{m}^{2}\right)
$$$$
\mathrm{d} n / \mathrm{d} t=\alpha_{\mathrm{n}}(1-n)-\beta_{\mathrm{n}} n(\mathrm{~Hz})
$$$$
\alpha_{\mathrm{n}} \quad=5.46 \times 104 \times\left(V_{\mathrm{m}}+0.0932\right) /\left(1-e^{(-0.0932-\mathrm{Vm}) / 0.00110}\right)(\mathrm{Hz})
$$$$
\beta_{\mathrm{n}}=9.71 \times 104 \times\left(-0.0760-V_{\mathrm{m}}\right) /\left(1-e^{(\mathrm{Vm}+0.0760) / 0.0105}\right)(\mathrm{Hz})
$$

Slow $K^{+}$current:

$$
\begin{gathered}
i_{K s}=g_{K s} s\left(V_{m}-E_{K}\right)\left(\mathrm{A} / \mathrm{m}^{2}\right) \\
\mathrm{d} s / \mathrm{d} t=\alpha_{\mathrm{s}}(1-s)-\beta_{\mathrm{n}} s(\mathrm{~Hz}) \\
\alpha_{\mathrm{s}}=8.34 \times 10^{3} \times\left(V_{\mathrm{m}}+0.0125\right) /\left(1-e^{(-0.0125-V \mathrm{~m}) / 0.0236}\right)(\mathrm{Hz}) \\
\beta_{\mathrm{s}}=5.05 \times 10^{3} \times\left(-0.0801-V_{\mathrm{m}}\right) /\left(1-e^{(V \mathrm{~m}+0.0801 / 0.0218}\right)(\mathrm{Hz})
\end{gathered}
$$

Leak current:

$$
i_{L}=g_{L}\left(V_{m}-E_{L}\right)\left(\mathrm{A} / \mathrm{m}^{2}\right)
$$

The nodal membrane parameters used in our model are listed in Table 4. 
TABLE 4

PARAMETER VALUES USED FOR NODES OF RANVIER IN THE AXON MODEL.

\begin{tabular}{|c|c|c|c|}
\hline Parameter & Value & Unit & Reference \\
\hline$c_{\text {node }}$ & 0.028 & $\mathrm{~F} / \mathrm{m}^{2}$ & [25] \\
\hline$p_{\mathrm{Na}}$ & $7.04 \cdot 10^{-5}$ & $\mathrm{~m} / \mathrm{s}$ & \multirow{7}{*}{ [27] } \\
\hline$\left[\mathrm{Na}_{\text {in }}\right]$ & 20 & $\mathrm{mM}$ & \\
\hline$\left[\mathrm{Na}_{\text {out }}\right]$ & 154 & $\mathrm{mM}$ & \\
\hline$E_{\mathrm{K}}$ & -0.084 & V & \\
\hline$g_{\mathrm{Kf}}$ & 300 & $\mathrm{~S} / \mathrm{m}^{2}$ & \\
\hline$g_{\mathrm{Ks}}$ & 600 & $\mathrm{~S} / \mathrm{m}^{2}$ & \\
\hline$E_{\mathrm{L}}$ & -0.084 & V & \\
\hline$g_{\mathrm{L}}$ & 400 & $\mathrm{~S} / \mathrm{m}^{2}$ & This study \\
\hline
\end{tabular}

[1]

The myelin conductance and capacitance are inversely related to the number of myelin lamella $n_{1}=D(1-g) / 2 d_{1}$ where each lamella is $d_{1}=24 \mathrm{~nm}$ thick:

$$
\begin{aligned}
& G_{\mathrm{my}}=\pi D L \frac{1+g}{2 n_{1}} g_{\mathrm{lam}} \\
& C_{\mathrm{my}}=\pi D L \frac{1+g}{2 n_{1}} c_{\mathrm{lam}}
\end{aligned}
$$

The axial conductance $g_{\mathrm{A}}$ between adjacent model segments is given by

$$
g_{\mathrm{A}}=\frac{\pi d^{2}}{2 L \rho_{\mathrm{a}}}
$$

The electrical parameters describing the myelinated fiber portions are summarized in Table 5.

TABLE 5.

PARAMETERS VALUES USED FOR AXIAL AND MYELINATED SEGMENTS OF THE AXON.

\begin{tabular}{|l|c|c|c|}
\hline \hline Parameter & Value & Unit & Reference \\
\hline$g_{\text {lam }}$ & 5 & $\mathrm{~S} / \mathrm{m}^{2}$ & \multirow{2}{*}[49]{} \\
\hline$c_{\text {lam }}$ & 0.0005 & $\mathrm{~F} / \mathrm{m}^{2}$ & \\
\hline$\rho_{\mathrm{A}}$ & 0.40 & $\Omega . \mathrm{m}$ & {$[50]$} \\
\hline$V_{\text {rest }}$ & -0.084 & $\mathrm{~V}$ & {$[27]$} \\
\hline \hline
\end{tabular}

The relation between $d, L$ and $D$ for the morphometric data used for construction of the axon fiber model are presented in Fig.7.
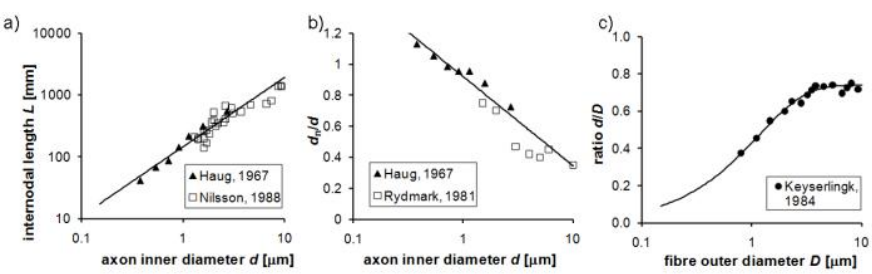

Fig. 7. Morphometric data used for construction of the fiber model. (a) Experimental relation between axon inner diameter, $d$, and internodal length $(L)$. (b) Dependence of normalized nodal diameter on axon inner diameter. (c) Variation of the ratio between $d$ and $D$ with fiber outer diameter. Drawn lines in the plots correspond to empirical fits to the data as described in equation 3 and 4 .

\section{REFERENCES}

A. L. Benabid, et al., "Deep brain stimulation of the subthalamic nucleus for the treatment of Parkinson's disease," Lancet Neurol, vol. 8, pp. 67-81, Jan 2009.

M. D. Johnson, et al., "Neuromodulation for brain disorders: challenges and opportunities," IEEE Trans Biomed Eng, vol. 60, pp. 610-24, Mar 2013.

S. Hemm and K. Wårdell, "Stereotactic implantation of deep brain stimulation electrodes: a review of technical systems, methods and emerging tools," Med Biol Eng Comput, vol. 48, pp. 611-24, Jul 2010.

C. C. McIntyre, et al., "Electric field and stimulating influence generated by deep brain stimulation of the subthalamic nucleus," Clin Neurophysiol, vol. 115, pp. 589-95, Mar 2004.

[5] A. M. Kuncel and W. M. Grill, "Selection of stimulus parameters for deep brain stimulation," Clin Neurophysiol, vol. 115, pp. 243141, Nov 2004.

[6] C. R. Butson, et al., "Patient-specific analysis of the volume of tissue activated during deep brain stimulation," Neuroimage, vol. 34, pp. 661-70, Jan 152007.

[7] M. Åström, et al., "Patient-Specific Model-Based Investigation of Speech Intelligibility and Movement during Deep Brain Stimulation," Stereotact Funct Neurosurg, vol. 88, pp. 224-233, May 122010.

[8] A. Mikos, et al., "Patient-specific analysis of the relationship between the volume of tissue activated during DBS and verbal fluency," Neuroimage, vol. 54 Suppl 1, pp. S238-46, Jan 2011.

[9] S. Miocinovic, et al., "Experimental and theoretical characterization of the voltage distribution generated by deep brain stimulation," Exp Neurol, vol. 216, pp. 166-76, Mar 2009.

[10] A. Chaturvedi, et al., "Patient-specific models of deep brain stimulation: influence of field model complexity on neural activation predictions," Brain Stimul, vol. 3, pp. 65-7, Apr 2010.

[11] S. Hemm, et al., "Deep brain stimulation in movement disorders: stereotactic coregistration of two-dimensional electrical field modeling and magnetic resonance imaging," J Neurosurg, vol. 103, pp. 949-55, Dec 2005.

[12] X. Vasques, et al., "Stereotactic model of the electrical distribution within the internal globus pallidus during deep brain stimulation," J Comput Neurosci, vol. 26, pp. 109-18, Feb 2009.

[13] M. Åström, et al., "Method for patient-specific finite element modeling and simulation of deep brain stimulation," Med Biol Eng Comput, vol. 47, pp. 21-8, Jan 2009.

[14] C. R. Butson and C. C. McIntyre, "Current steering to control the volume of tissue activated during deep brain stimulation," Brain Stimul, vol. 1, pp. 7-15, Jan 2008.

[15] H. C. Martens, et al., "Spatial steering of deep brain stimulation volumes using a novel lead design," Clin Neurophysiol, vol. 122, pp. 558-66, Mar 2011.

[16] C. R. Butson and C. C. McIntyre, "Role of electrode design on the volume of tissue activated during deep brain stimulation," J Neural Eng, vol. 3, pp. 1-8, Mar 2006.

[17] C. Schmidt, et al., "Influence of uncertainties in the material properties of brain tissue on the probabilistic volume of tissue activated," IEEE Trans Biomed Eng, vol. 60, pp. 1378-87, May 2013.

[18] A. L. Benabid, et al., "Chronic electrical stimulation of the ventralis intermedius nucleus of the thalamus as a treatment of movement disorders," J Neurosurg, vol. 84, pp. 203-14, Feb 1996. C. C. McIntyre and P. J. Hahn, "Network perspectives on the mechanisms of deep brain stimulation," Neurobiol Dis, vol. 38, pp. 329-37, Jun 2010

[20] J. B. Ranck, Jr., "Which elements are excited in electrical stimulation of mammalian central nervous system: a review," Brain research, vol. 98, pp. 417-40, Nov 211975.

[21] J. Holsheimer, "Principles of Neurostimulation," in Electric stimulation and the relief of pain. vol. 15, B. A. Simpson, Ed., ed Amsterdam, The Netherlands: Elsevier Health Sciences, 2003, pp. 17-36.

[22] G. Weiss, "Sur la possibilité de render comparables entre eux les appareils servant à l'excitation électrique.," Arch Ital Biol, vol. 35, p. $35: 46,1901$. 
[23] A. Chaturvedi, et al., "Current steering to activate targeted neural pathways during deep brain stimulation of the subthalamic region," Brain Stimul, vol. 5, pp. 369-77, Jul 2012.

[24] S. N. Sotiropoulos and P. N. Steinmetz, "Assessing the direct effects of deep brain stimulation using embedded axon models," $J$ Neural Eng, vol. 4, pp. 107-19, Jun 2007.

[25] W. A. Wesselink, et al., "A model of the electrical behaviour of myelinated sensory nerve fibres based on human data," Med Biol Eng Comput, vol. 37, pp. 228-35, Mar 1999.

[26] A. G. Richardson, et al., "Modelling the effects of electric fields on nerve fibres: influence of the myelin sheath," Med Biol Eng Comput, vol. 38, pp. 438-46, Jul 2000.

[27] J. R. Schwarz, et al., "Action potentials and membrane currents in the human node of Ranvier," Pflugers Arch, vol. 430, pp. 283-92, Jun 1995.

[28] H. Haug, "[Length of internodes of myelinated fibers in the region of the optic cortex of the adult cat]," Z Zellforsch Mikrosk Anat, vol. 83, pp. 265-78, 1967.

[29] I. Nilsson and C. H. Berthold, "Axon classes and internodal growth in the ventral spinal root L7 of adult and developing cats," J Anat, vol. 156, pp. 71-96, Feb 1988.

[30] M. Rydmark, "Nodal axon diameter correlates linearly with internodal axon diameter in spinal roots of the cat," Neurosci Lett, vol. 24, pp. 247-50, Jul 171981.

[31] D. Graf von Keyserlingk and U. Schramm, "Diameter of axons and thickness of myelin sheaths of the pyramidal tract fibres in the adult human medullary pyramid," Anat Anz, vol. 157, pp. 97-111, 1984

[32] J. Holsheimer, et al., "Identification of the target neuronal elements in electrical deep brain stimulation," European Journal of Neuroscience, vol. 12 pp. 4573-4577, 2000.

[33] J. Holsheimer, et al., "Chronaxie calculated from current-duration and voltage-duration data," Journal of Neuroscience Methods, vol. 97, pp. 45-50, 2000.

[34] A. M. Kuncel, et al., "A method to estimate the spatial extent of activation in thalamic deep brain stimulation," Clin Neurophysiol, vol. 119, pp. 2148-58, Sep 2008.

[35] F. Aboitiz, et al., "Fiber composition of the human corpus callosum," Brain Res, vol. 598, pp. 143-53, Dec 111992.

[36] C. Schmidt and U. van Rienen, "Modeling the field distribution in deep brain stimulation: the influence of anisotropy of brain tissue," IEEE Trans Biomed Eng, vol. 59, pp. 1583-92, Jun 2012.

[37] D. K. Cheng, Field and wave electromagnetics. Reading, Mass.: Addison-Wesley, 1989.

[38] C. R. Butson and C. C. McIntyre, "Tissue and electrode capacitance reduce neural activation volumes during deep brain stimulation," Clinical neurophysiology : official journal of the International Federation of Clinical Neurophysiology, vol. 116, pp. 2490-500, Oct 2005.

[39] C. C. McIntyre, et al., "Modeling the excitability of mammalian nerve fibers: influence of afterpotentials on the recovery cycle," $J$ Neurophysiol, vol. 87, pp. 995-1006, Feb 2002.

[40] B. Madler and V. A. Coenen, "Explaining clinical effects of deep brain stimulation through simplified target-specific modeling of the volume of activated tissue," AJNR Am J Neuroradiol, vol. 33, pp. 1072-80, Jun 2012.

[41] A. Mathai, et al., "More than meets the eye-myelinated axons crowd the subthalamic nucleus," Mov Disord, vol. 28, pp. 1811-5, Nov 2013.

[42] S. Hemm, et al., "Co-registration of stereotactic MRI and isofieldlines during deep brain stimulation," Brain Res Bull, vol. 68, pp. 59-61, Dec 152005.

[43] K. Wårdell, et al., "Patient-Specific Brain Modelling for Deep Brain Stimulation Simulation," in 6th International IEEE EMBS Conference on Neural Engineering proceedings, page 148-151, 2013.

[44] M. Åström, et al., "Influence of heterogeneous and anisotropic tissue conductivity on electric field distribution in deep brain stimulation," Med Biol Eng Comput, vol. 50, pp. 23-32, Jan 2012.

[45] B. Howell, et al., "Influences of interpolation error, electrode geometry, and the electrode-tissue interface on models of electric fields produced by deep brain stimulation," IEEE Trans Biomed Eng, vol. 61, pp. 297-307, Feb 2014.
T. C. Zhang and W. M. Grill, "Modeling deep brain stimulation: point source approximation versus realistic representation of the electrode," J Neural Eng, vol. 7, p. 066009, Dec 2010.

[47] G. Walckiers, et al., "Influence of the implanted pulse generator as reference electrode in finite element model of monopolar deep brain stimulation," J Neurosci Methods, vol. 186, pp. 90-6, Jan 30 2010.

[48] P. F. Grant and M. M. Lowery, "Electric field distribution in a finite-volume head model of deep brain stimulation," Med Eng Phys, vol. 31, pp. 1095-103, Nov 2009.

[49] A. F. Huxley and R. Stampfli, "Evidence for saltatory conduction in peripheral myelinated nerve fibres," J Physiol, vol. 108, pp. 31539, May 151949.

[50] K. R. Foster, et al., "The electrical resistivity of cytoplasm," Biophys J, vol. 16, pp. 991-1001, Sep 1976.

Mattias Åström received a M.Sc. degree in information technology and biomedical engineering in 2004, and a Ph.D. degree in Biomedical Instrumentation from Linköping Institute of Technology, Linköping, Sweden in 2011. Currently he holds a position as a research scientist at Sapiens Steering Brain Stimulation where he develops concepts for image-guided tuning of DBS. He is also an adjunct lecturer in biomedical engineering at Linköping University. His present research interest is related to modeling, simulation, and visualization deep brain stimulation and its translation into clinical applications.

Elin Diczfalusy received a M.Sc. degree in engineering biology in 2009, and a Licenciate degree in Biomedical Instrumentation from Linköping Institute of Technology, Linköping University, Sweden, in 2012. Her research interests include biomedical simulations and visualizations within the area of neuroscience and neuro-engineering.

Hubert Martens received a M.Sc. degree in physics in 1996, and a Ph.D. degree (cum laude) in physics from Leiden University, Leiden, The Netherlands in 2000. Hubert is a co-founder of Sapiens Steering Brain Stimulation B.V. and as VP Product Development at Sapiens he is responsible for the realization of Sapiens' portfolio of deep brain stimulation solutions. He is driven to advance neuromodulation technologies that enable tailored therapies for patients suffering from brain disorders.

Karin Wårdell received a M.Sc. degree in electrical engineering and technical physics in 1987, and a Ph.D. degree in Biomedical Instrumentation from Linköping Institute of Technology, Linköping, Sweden in 1994. She is currently Professor in biomedical engineering at Linköping University. Her present research interest include neuro-engineering related to brain stimulation, modeling and simulation, navigation, biomedical optics and microcirculation, and its translation into clinical applications. She is a member of the IEEE Engineering in Medicine and Biology Society and Biomedical Engineering Society, and a Fellow at the International Academy of Medical and Biological Engineering and the European Alliance of Medical and Biological Engineering and Sciences. 